\title{
Treatment with 4-aminopyridine improves upper limb tremor of a patient with multiple sclerosis: a video case report
}

Multiple Sclerosis Journal

19(4) 506-508

(C) The Author(s) 2012

Reprints and permissions:

sagepub.co.uk/journalsPermissions.nav DOI: 10.1 I $77 / 1352458512461394$

msj.sagepub.com

@SAGE

\author{
Roman Schniepp ${ }^{1,2}$, Veronika Jakl', Max Wuehr², Joachim \\ Havla',3, Tanja Kümpfel',3, Marianne Dieterich', Michael Strupp ${ }^{1,2}$ \\ and Klaus Jahn ${ }^{1,2}$
}

\begin{abstract}
The reversible potassium channel blocker 4-aminopyridine is effective in the treatment of numerous cerebellar dysfunctions, such as episodic ataxia type 2 and downbeat nystagmus syndrome. In 20II, its sustained release form, dalfampridine, was admitted in Europe for the treatment of walking difficulties in patients with multiple sclerosis (MS). Here we report the case of a 44-year old patient with a progressive MS whose upper limb tremor was markedly reduced under treatment with 4-aminopyridine, as documented in a Tremor Activities of Daily Living questionnaire and in the 9-Hole Peg test. Hand accelerations decreased in the left hand from $10.9 \mathrm{~m} / \mathrm{sec}^{2}$ to $2.2 \mathrm{~m} / \mathrm{sec}^{2}$ and in the right hand from $4.2 \mathrm{~m} / \mathrm{sec}^{2}$ to $0.9 \mathrm{~m} / \mathrm{sec}^{2}$.

This case report indicates for the first time that 4-aminopyridine might be effective in the symptomatic treatment of tremor entities in patients with MS. The finding calls for further prospective studies to determine the usefulness of 4-aminopyridine or its sustained-release form dalfampridine in treating patients with tremor and MS.
\end{abstract}

\section{Keywords}

Postural tremor, 4-aminopyridine, cerebellar ataxia, multiple sclerosis, case study, tremor therapy

Date received: 20th June 2012; accepted: 16th August 2012

\section{Introduction}

In March 2010, the US Food and Drug Administration (FDA) approved the sustained release form of 4-aminopyridine (4-AP), Fampyra ${ }^{\circledR}$ (dalfampridine), for the treatment of walking difficulties in patients with multiple sclerosis (MS). This drug improves gait speed in approximately $40 \%$ of MS patients. ${ }^{1}$ The active ingredient, 4-AP, is a reversible potassium-channel blocker used in experimental neurophysiology for decades; over the last years, a number of studies have shown that 4-AP has the ability to improve specific cerebellar dysfunctions. In episodic ataxia type 2 (EA2), it prevents attacks and improves the patient's quality of life. ${ }^{2}$ In two separate cases with EA2, gait variability became reduced and the subjective fall risk decreased during treatment with 4-AP. ${ }^{3}$ In patients with downbeat nystagmus syndrome secondary to cerebellar floccular dysfunction, the intensity of nystagmus was reduced during treatment with 4-AP. ${ }^{4}$

\section{Case report}

Here we report a single case in which there was a positive effect of 4-AP treatment on upper limb tremors. A 44-yearold woman with a progressive MS presented to the hospital with an Expanded Disability Status Scale (EDSS) score of 6.5. Besides spastic-ataxic walking impairments, her major presentations were the presence of a postural and intention tremor of the upper extremities and the trunk, leading to a severe reduction of upper limb function, e.g. while brushing her teeth or drinking a glass of water.

\footnotetext{
'Department of Neurology, University of Munich, Germany.

${ }^{2}$ German Vertigo/Dizziness Center; Integriertes Forschungs- und Behandlungszentrum, IFBLMU

${ }^{3}$ Institute of Clinical Neuroimmunology, University of Munich, Germany.
}

\section{Corresponding author:}

Roman Schniepp, University Hospital Munich, Campus Grosshadern, Department of Neurology, Marchioninistrasse 15, 8 I 377 Munich, Germany. Email: roman.schniepp@med.uni-muenchen.de 

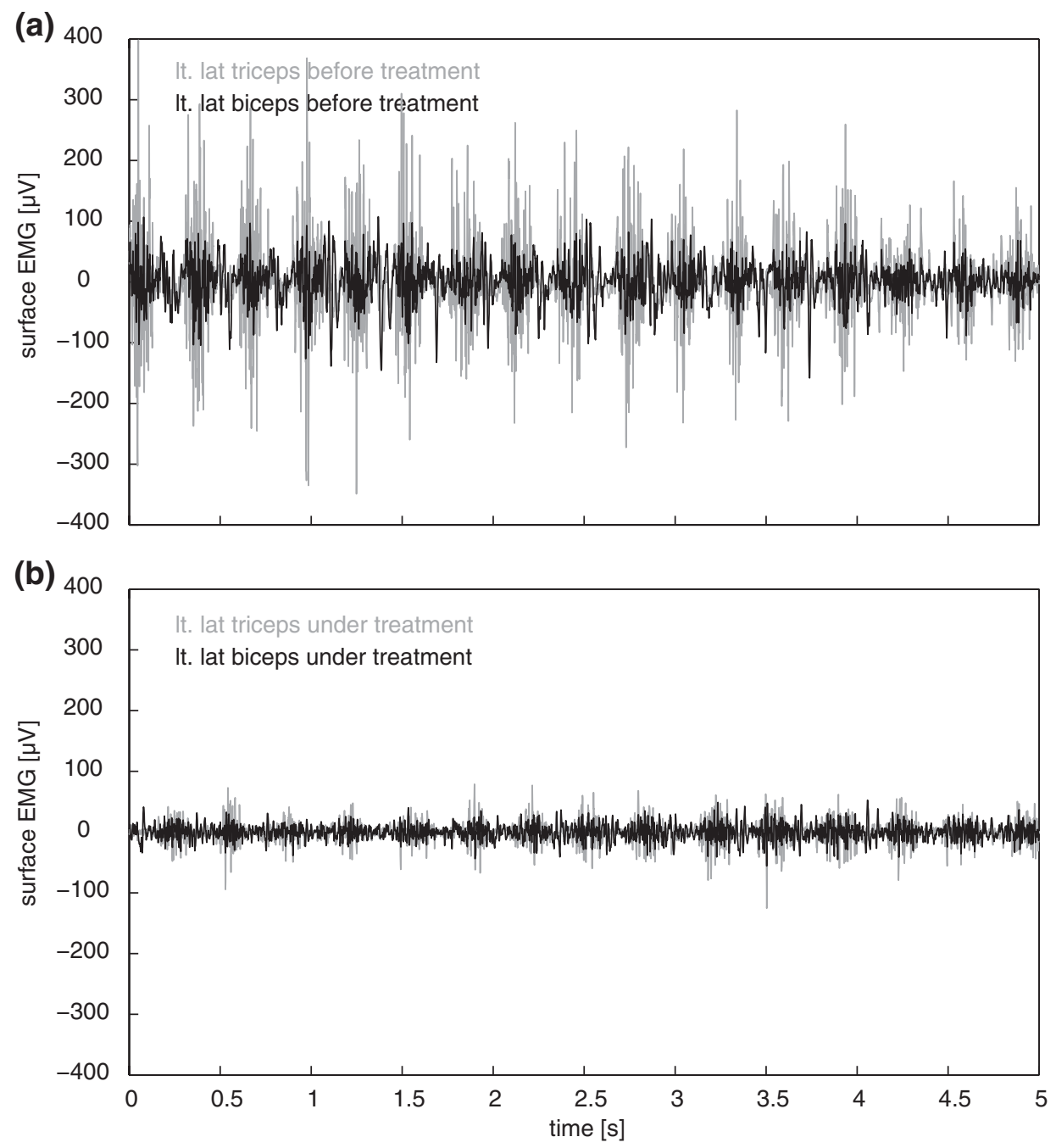

Figure I. Surface EMG recordings of the left proximal muscles.

(a) Compounding action potentials of the left arm over a 5-s time period, under the condition of holding, before 4-AP therapy;

(b) Compounding action potentials of the left arm over a 5-s time period, under the condition of holding, under the influence of 4-AP therapy. 4-AP: 4-aminopyridine; EMG: electromyography.

Assessment of the upper limb function included the 9-Hole Peg test. The time for completion of this test was $75 \mathrm{~s}$, with the right hand. The test could not be completed by the patient with her non-dominant left hand. On a questionnaire-based tremor scale of activities of daily living, she rated 89 out of a maximum of 100 points. Tremor analysis using the Noraxon ${ }^{\circledR}$ system was performed using a surface electromyography (EMG) over bilateral antagonistic muscles, as well as 3-dimensional (3-D) accelerometers that were mounted bilaterally, at the dorsal surface of both hands. The accelerometer and EMG recordings were performed under the condition of holding the upper limbs against gravity, in a sitting position. Tremor intensity was calculated using a standard 3-D root mean square analysis. Her tremor intensities (hand accelerations) were $10.89 \mathrm{~m} / \mathrm{s}^{2}$ for the left hand and $4.23 \mathrm{~m} / \mathrm{s}^{2}$ for the right hand, with a frequency of 3.0 to 3.4 $\mathrm{s}^{-1}$ (see Video 1, in the supplemental data).

The patient was symptomatically treated with 4-AP (5 mg t.i.d.). Only 24 hours after the first administration, the patient reported there had been an improvement of her arm and hand tremors. Subjective scoring of the tremor-related activities of daily living improved from 89 to 77 points. With her dominant right hand, she completed the 9-Hole Peg test within 56s (a reduction of $19 \mathrm{~s}$ ). Completion of the 9-Hole Peg test with her left hand was still not possible. After $62 \mathrm{~h}$ of treatment, the accelerometer recordings and video analysis were repeated, revealing a marked decrease in tremor intensity (see Video 1, supplemental data). Hand acceleration decreased by approximately $80 \%$ (left hand: reduction from $10.9 \mathrm{~m} / \mathrm{s}^{2}$ to $2.2 \mathrm{~m} / \mathrm{s}^{2}$; right hand: reduction from $4.2 \mathrm{~m} / \mathrm{s}^{2}$ to $0.9 \mathrm{~m} / \mathrm{s}^{2}$ ). Surface-EMG recordings showed a reduction of both distal and proximal arm muscle activity (for proximal muscles, see Figure 1). The frequency of the tremor remained unaffected.

Besides the effect on the hand tremors, the patient's gait performance (gait speed, stride length, gait variability) improved under 4-AP treatment (data not shown). 
After a follow-up period of 4 weeks, all improvements in subjective, clinical and neurophysiological measurements remained stable (data not shown). The patient is continuing the 4-AP treatment, which has lasted for more than 18 months now. She has refused to switch her medication from 4-AP to dalfampridine, citing both its beneficial effects and an absence of side effects.

\section{Discussion}

The symptomatic treatment of MS patients with cerebellar tremor remains a challenge for clinicians. Pharmacotherapeutic strategies are described in several small, short-duration studies ${ }^{5,6}$ but evidence is conflicting. Deep brain stimulation, mainly of the ventral intermediate thalamic nuclei, may obtain tremor reduction, but this is associated with potentially serious side effects. ${ }^{7}$

This case report indicates for the first time the usefulness of 4-AP in improving the upper limb tremor of a MS patient. Thus, besides its positive effect on walking performance in MS patients, 4-AP may also be considered as a new option for the symptomatic treatment of tremor in patients with MS.

Aminopyridines were previously tested in the symptomatic treatment of tremor patients. A prospective, doubleblinded cross-over trial with 3,4-diaminopyridine in 19 patients with essential tremor did not show any positive effects. ${ }^{8}$ Our case report indicates that aminopyridines may be effective in treating MS tremor, secondary to pathologies of the cerebellum. Our patient showed a high burden of disease within the cerebellum and cerebellar peduncles (Figure 2). Clinical testing revealed a marked cerebellar ataxia and the criteria for a cerebellar tremor (low frequency, postural and intention tremor) were fulfilled.

Supported by animal studies, the putative mode of action of 4-AP on cerebellar functions is thought to be an improvement of Purkinje cell function. The drug 4-AP improves the excitability and the precision of cerebellar Purkinje cells. ${ }^{9}$ Moreover, 4-AP reduces the excitability of olivary neurons, at least in the guinea pig, which in turn are thought to be the rhythm pacemakers in the harmaline-induced tremor model. ${ }^{10}$

Taking these findings into account, one can argue that 4-AP may have the potential to improve any tremor entities (a)

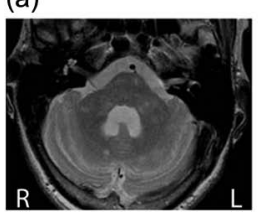

(b)

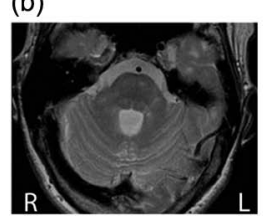

(c)

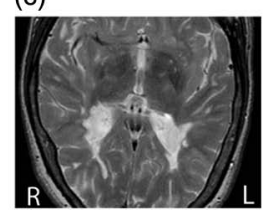

Figure 2. T2 sequences of the brain of the patient, prior to treatment, using a Siemens ${ }^{\circledR}$ MRI Scanner at I.5 Tesla.

(a) T2 $(3 \mathrm{~mm})$ sequence of the cerebellum, inferior cerebellar peduncles and brainstem

(b) T2 $(3 \mathrm{~mm})$ sequence of the cerebellum, middle cerebellar peduncles and brainstem

(c) T2 $(3 \mathrm{~mm})$ sequence of the inferior thalamus

MRI: magnetic resonance imaging; that are linked to cerebellar dysfunctions. This further implies that our observation should encourage us to further investigate the usefulness of 4-AP, or its sustained release formulation dalfampridine; in a systemic, controlled trial in patients with MS tremor, especially when cerebellar symptoms are present.

\section{Funding}

This work was supported by the German research foundation Deutsche Forschungsgemeinschaft (grant number DFG JA 1087/1-1) and the Federal Ministry of Education and Research Bundesministerium für Bildung und Forschung, BMBF 01EO0901.

\section{Conflict of interest}

The authors declare that there are no conflicts of interest.

\section{Acknowledgements}

The authors thank Judy Benson and Cauchy Pradhan for editing the article content.

\section{Contributorship statement}

1. Research project: A. Conception B. Organization C. Execution 2. Manuscript: A. Writing of the first draft $B$. Review and Critique R. Schniepp 1ABC, 2AB, V. Jakl 1B, 2B, M. Wuehr 1A, 2B, J. Havla 1C, 2B, T. Kümpfel 1C, 2B, M. Dieterich 1AB, 2B, M. Strupp 1A, 2B, K. Jahn 1AB, 2AB

\section{References}

1. Goodman AD, Brown TR, Edwards KR, et al. A phase 3 trial of extended release oral dalfampridine in multiple sclerosis. Ann Neurol 2010; 68: 494-502.

2. Strupp M, Kalla R, Claassen J, et al. A randomized trial of 4-aminopyridine in EA2 and related familial episodic ataxias. Neurology 2011; 77: 269-275.

3. Schniepp R, Wuehr M, Ackl N, et al. 4-aminopyridine improves gait variability in cerebellar ataxia due to CACNA 1A mutation. J Neurol 2011; 258: 1708-1711.

4. Strupp M, Schuler O, Krafczyk S, et al. Treatment of downbeat nystagmus with 3,4-diaminopyridine: A placebo-controlled study. Neurology 2003; 61: 165-170.

5. Feys P, D'hooghe MB, Nagels G, et al. The effect of levetiracetam on tremor severity and functionality in patients with multiple sclerosis. Mult Scler 2009; 15: 371-378.

6. Naderi F, Javadi SA, Motamedi M, et al. The efficacy of primidone in reducing severe cerebellar tremors in patients with multiple sclerosis. Clin Neuropharmacol 2012; Vol: 1243-1251.

7. Hassan A, Ahlskog JE, Rodriguez M, et al. Surgical therapy for multiple sclerosis tremor: A 12-year follow-up study. Eur J Neurol 2012; 19: 764-768.

8. Lorenz D, Hagen K, Ufer M, et al. No benefit of 3,4-diaminopyridine in essential tremor: A placebo-controlled crossover study. Neurology 2006; 66: 1753-1755.

9. Alvina $\mathrm{K}$ and Khodakhah $\mathrm{K}$. The therapeutic mode of action of 4-aminopyridine in cerebellar ataxia. J Neurosci 2010; 30 : 7258-7268.

10. Wilms H, Sievers J and Deuschl G. Animal models of tremor. Mov Disord 1999; 14: 557-571. 